\title{
Color screening in lattice QCD and at weak coupling
}

\author{
Johannes Heinrich Weber* [TUMQCD collaboration] \\ Technische Universität München \\ E-mail: johannes.weberetum.de
}

\begin{abstract}
We study spatial correlation functions of static quark-antiquark pairs in QCD with $2+1$ flavors in order to better understand color screening at high temperatures. We performed lattice simulations in a wide temperature window $115 \mathrm{MeV} \lesssim T \lesssim 5.8 \mathrm{GeV}$ using the using the highly improved staggered quark (HISQ) action, the tree-level improved Symanzik gauge action and several lattice spacings to control discretization effects. By comparing our lattice results to analytic calculations at weak coupling as well as to the zero temperature result on the static energy we demonstrate that color screening sets in at distances $r T \simeq 0.25$. We also conclude that in the distance regime $0.25<r T<0.6$ the weak-coupling approach provides an adequate description of color screening.
\end{abstract}

Critical Point and Onset of Deconfinement - CPOD2017

7-11 August, 2017

The Wang Center, Stony Brook University, Stony Brook, NY

\footnotetext{
*Speaker.
} 


\section{Introduction}

It is known from quantum field theoretical studies as well as from heavy-ion collision experiments at RHIC, GSI or LHC and astronomical observations of neutron stars that strongly interacting matter exhibits very different properties depending on the temperature and the baryon density. For sufficiently small values of the baryochemical potential a high temperature phase called quark-gluon-plasma has been established. Distinctive properties of quark-gluon-plasma are color screening, deconfinement and restoration of chiral symmetry, cf. recent reviews $[1,2]$.

In the limit of vanishing sea quark masses or in the limit of zero flavors of sea quarks, the transition between quark-gluon-plasma and the vacuum is sharp. For pure gauge theory and the gauge group $\mathrm{SU}\left(N_{c}\right)$, the Polyakov loop is the order parameter of a deconfinement transition, which is related to the spontaneous breaking of the $\mathrm{Z}\left(N_{c}\right)$ center symmetry of the Yang-Mills vacuum. Since the center symmetry is broken by the presence of sea quarks with sufficiently small quark masses already in the hadronic phase, the Polyakov loop does not play the role of an order parameter in full QCD. For massless sea quarks, the chiral susceptibility is the order parameter of the chiral phase transition. For sufficiently large sea quark masses, the chiral symmetry is already broken explicitly by the quark masses and the chiral susceptibility does not play the role of an order parameter in full QCD. In an intermediate range of sea quark masses that includes the physical point, neither is an order parameter and the QCD transition is a crossover. Nevertheless, would-be order parameters still provide insight into the mechanisms of breaking the associated approximate symmetries.

Polyakov loops and correlators of Polyakov loops are observables that are sensitive to color screening. After proper renormalization the Polyakov loop correlator $C_{P}$ can be regarded as the correlator of a heavy quark-antiquark pair in the limit of infinite heavy quark mass. $C_{P}$ is related to the free energy of a static $Q \bar{Q}$ pair separated by $r$, namely $C_{P}(r, T)=\exp \left[-F_{Q \bar{Q}}(r, T) / T\right]$. In the following we report on preliminary results of a forthcoming extensive analysis of Polyakov loop correlators in lattice QCD with 2+1 flavors of sea quarks [3].

\section{Setup}
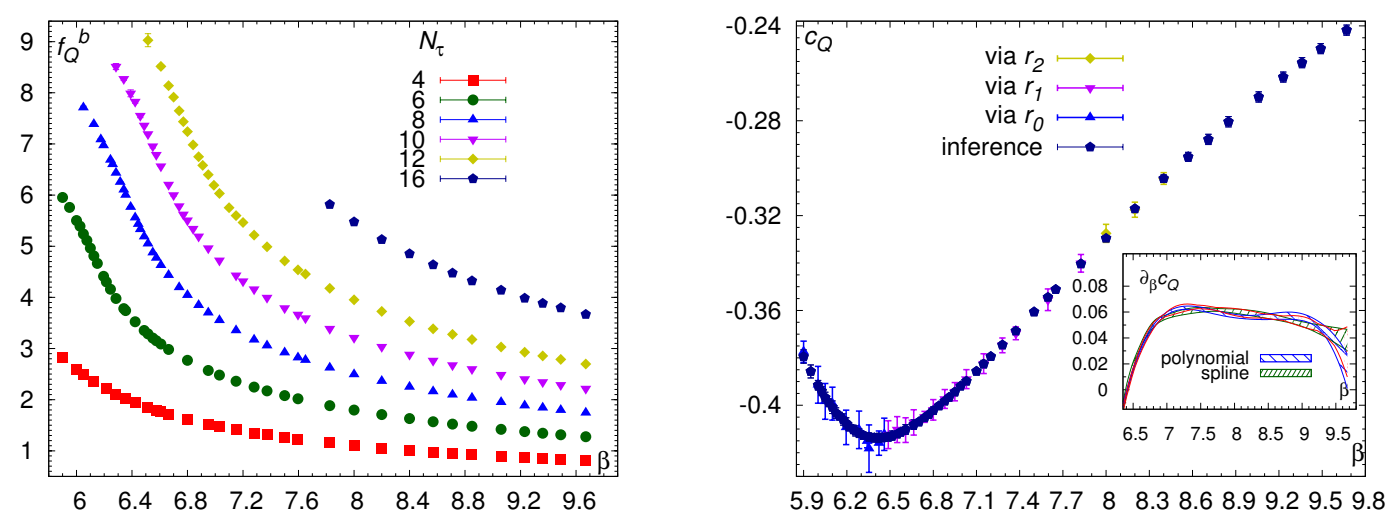

Figure 1: Combined TUMQCD and HotQCD 2+1 flavor gauge ensembles in terms of the bare free energy $f_{Q}^{\mathrm{b}}$ (left). The renormalization constant $c_{Q}$ from direct renormalization supersedes the old result [6] (right). 
We perform simulations of QCD with 2+1 flavors of sea quarks and calculate the Polyakov loop correlator as well as the color-singlet correlator of a static $Q \bar{Q}$ pair in Coulomb gauge. For our simulations we use the MILC code. We employ the highly improved staggered quark (HISQ) action and tree-level improved Symanzik gauge action. The gauge ensembles have been generated by the HotQCD and TUMQCD collaborations $[3,4,5,6]$. A detailed account of all ensembles is given in [3]. We use lattices with extensions $N_{\sigma}{ }^{3} \times N_{\tau}$ or $N_{\sigma}{ }^{2} \times N_{z} \times N_{\tau}$. For the temporal extent we use $N_{\tau}=16,12,10,8,6$ and 4 , for the spatial extent we choose $N_{\sigma} / N_{\tau}=4$ and 6 . We also use a few ensembles with $N_{z}=2 N_{\sigma}$. We set the strange quark mass $m_{s}$ to its physical value and use a degenerate light quark doublet with $m_{l}=m_{s} / 20$ or $m_{l}=m_{s} / 5$. These choices correspond in the continuum limit to pion masses of $m_{\pi} \approx 160$ or $320 \mathrm{MeV}$ respectively. We fix the strange quark mass in terms of the hypothetical $\eta_{s \bar{s}}$ meson, $m_{s \bar{s}} \approx 695 \mathrm{MeV}$, or if determination on $T=0$ lattices with the same parameters is not possible, through the lattice mass renormalization function determined in [5]. We fix the lattice spacing using the $r_{1}$ scale and the non-perturbative $\beta$ function defined in [7]. The scale parameters $r_{0}, r_{1}$ and $r_{2}$ are defined in terms of the $T=0$ static energy $V_{S}$ and the corresponding force,

$$
\left.r^{2} \frac{d V_{S}}{d r}\right|_{r=r_{0}}=1.65,\left.\quad \quad r^{2} \frac{d V_{S}}{d r}\right|_{r=r_{1}}=1,\left.\quad r^{2} \frac{d V_{S}}{d r}\right|_{r=r_{2}}=\frac{1}{2} .
$$

We obtain $r_{1}$ from $r_{0}$ on coarser lattices and from $r_{2}$ on finer lattices. The bare gauge couplings $\beta=10 / g_{0}^{2}$ that we use are in the range of $5.9 \leq \beta \leq 9.67$, which corresponds to lattice spacings of $a \approx 0.0085$ to $0.25 \mathrm{fm}$. We have about 30 different values of $\beta$ available for each $N_{\tau}$ except $N_{\tau}=16$. A chart of the combined TUMQCD and HotQCD ensembles is shown in the left panel of Fig. 1. Therefore, our simulations span the temperature range between $T \approx 115$ and $5814 \mathrm{MeV}$. The full temperature interval between 134 and $2326 \mathrm{MeV}$ is covered by at least four different $N_{\tau}$.

All of our $T>0$ ensembles with $m_{l}=m_{s} / 5$ have temperatures of at least $T \approx 350 \mathrm{MeV}$ such that the quark mass dependence is very mild. We observe that Polyakov loops and the correlators in ensembles with different quark masses are numerically compatible within uncertainties, although most $m_{l}=m_{s} / 5$ ensembles have smaller statistical uncertainties. If both are available we choose the ensemble with better signal-to-noise ratio and combine ensembles with different $m_{l}$ into a common data set. We use the same $r_{1}$ scale independent of the quark mass, since the $r_{1}$ for both setups is known to differ by only about $1 \%$ [7], which is hardly relevant for our study.

We calculate the bare Wilson line $W$, the bare Polyakov loop $P$ and the corresponding bare Polyakov loop expectation value $L^{\mathrm{b}}$ as

$$
W\left(N_{\tau}, \boldsymbol{x}\right)=\prod_{\tau=1}^{a N_{\tau}} U^{0}(\tau, \boldsymbol{x}), \quad P\left(N_{\tau}, \boldsymbol{x}\right)=\frac{1}{3} \operatorname{Tr}_{c} W\left(N_{\tau}, \boldsymbol{x}\right), \quad L^{\mathrm{b}}\left(\beta, N_{\tau}\right)=\left\langle P\left(N_{\tau}, \boldsymbol{x}\right)\right\rangle_{\beta},
$$

where we average over the full volume before taking the ensemble average denoted through the angle brackets $\langle\ldots\rangle_{\beta}$. $L^{\mathrm{b}} \equiv L_{F}^{\mathrm{b}}$ is the Polyakov loop in the fundamental representation. We renormalize the Polyakov loop through $L\left(\beta, N_{\tau}\right)=\exp \left[a C_{Q}(\beta) N_{\tau}\right] L^{\mathrm{b}}\left(\beta, N_{\tau}\right)$, where $C_{Q}$ is a renormalization constant that removes the self-energy divergence due to the gauge links $U^{0}$. We obtain $C_{Q}$ from the $T=0$ static energy $V_{S}$ at $r=r_{0}, r_{1}$ or $r_{2}$ for $\beta \leq 8.4$ and extend it to $\beta>8.4$ using direct renormalization as described in [6]. We require only one iteration of direct renormalization 
owing to the enlarged data set. In the right panel of Fig. 1 we show that the uncertainties of $C_{Q}$ for $7.03<\beta<8.85$ are much reduced due to the inference between six different $N_{\tau}$ in the direct renormalization procedure thanks to the new $T=0$ lattices with $\beta \geq 8.0$ and thanks to the new high temperature lattices with $N_{\tau}>8$ and $\beta>8$.4. We obtain the free energy of an isolated static quark as $F_{Q}(T, a)=-T \ln L\left(\beta, N_{\tau}\right)$. In free energies we trade $\beta$ and $N_{\tau}$ for the physical temperature $T=1 /\left(a N_{\tau}\right)$ and the lattice spacing $a$. The expectation values of the bare correlators are

$$
\begin{aligned}
& C_{P}^{\mathrm{b}}\left(\beta, N_{\tau}, r\right)=\left\langle P\left(N_{\tau}, \boldsymbol{x}\right) P^{\dagger}\left(N_{\tau}, \boldsymbol{x}+\boldsymbol{r}\right)\right\rangle_{\beta}, \\
& C_{S}^{\mathrm{b}}\left(\beta, N_{\tau}, r\right)=\frac{1}{3}\left\langle\operatorname{Tr}_{c}\left\{W\left(N_{\tau}, \boldsymbol{x}\right) W^{\dagger}\left(N_{\tau}, \boldsymbol{x}+\boldsymbol{r}\right)\right\}\right\rangle_{\beta} .
\end{aligned}
$$

For Eq. (2.4) we have fixed Coulomb gauge and the trace $\operatorname{Tr}_{c}\{\ldots\}$ runs over all color indices. We average the correlators over the full volume before taking the ensemble average. We may interpret Eq. (2.4) as a static meson correlation function at Euclidean time $\tau=1 / T$ with a separation $r$ between the quark and antiquark. In the infinite volume limit the static $Q \bar{Q}$ decouple and the correlators approach $\left|L^{\mathrm{b}}\left(\beta, N_{\tau}\right)\right|^{2}$. The self-energy divergences due to the gauge links $U^{0}$ contributing to the Polyakov loop and the correlators cancel exactly in the ratios

$$
\begin{aligned}
& C_{P}^{\mathrm{sub}}\left(\beta, N_{\tau}, r\right)=\left\langle\frac{\left\langle P\left(N_{\tau}, \boldsymbol{x}\right) P^{\dagger}\left(N_{\tau}, \boldsymbol{x}+\boldsymbol{r}\right)\right\rangle_{\boldsymbol{x}}}{\left\langle P\left(N_{\tau}, \boldsymbol{x}\right)\right\rangle_{\boldsymbol{x}}^{2}}\right\rangle_{\beta}, \\
& C_{S}^{\mathrm{sub}}\left(\beta, N_{\tau}, r\right)=\frac{1}{3}\left\langle\frac{\left\langle\operatorname{Tr}_{c}\left\{W\left(N_{\tau}, \boldsymbol{x}\right) W^{\dagger}\left(N_{\tau}, \boldsymbol{x}+\boldsymbol{r}\right)\right\}\right\rangle_{\boldsymbol{x}}}{\left\langle P\left(N_{\tau}, \boldsymbol{x}\right)\right\rangle_{\boldsymbol{x}}^{2}}\right\rangle_{\beta},
\end{aligned}
$$

which are do not require renormalization. Here we explicitly separate the volume average $\langle\ldots\rangle_{\boldsymbol{x}}$ and the ensemble average $\langle\ldots\rangle_{\beta}$, taking the ratios along the molecular dynamics history to benefit from partial cancellation of statistical fluctuations between the correlators for large $Q \bar{Q}$ separations and the Polyakov loops. We define subtracted free energies in terms of these ratios,

$$
F_{Q \bar{Q}}^{\mathrm{sub}}(r, T, a)=-T \ln C_{P}^{\mathrm{sub}}\left(\beta, N_{\tau}, r\right), \quad F_{S}^{\mathrm{sub}}(r, T, a)=-T \ln C_{S}^{\mathrm{sub}}\left(\beta, N_{\tau}, r\right) .
$$

By adding $2 F_{Q}(T, a)$ we obtain renormalized free energies $F_{Q \bar{Q}}=F_{Q \bar{Q}}^{\text {sub }}+2 F_{Q}$ and $F_{S}=F_{S}^{\text {sub }}+2 F_{Q}$. We denote any free energies in the following just by $F$ if no distinction is required and write for $F$ in units of the temperature $f=F / T$. Similarly we write for the renormalization constant times the lattice spacing $c_{Q}=a C_{Q}$, cf. Fig. 1 .

\section{Lattice results}

In this section we discuss the main results of our lattice calculation. The free energies $F$ are available only for discrete sets of temperatures that are different for each $N_{\tau}$. Moreover, for fixed temperature $T=1 /\left(a N_{\tau}\right)$ the available separations $r$ or $r T$ are not the same for data with different $N_{\tau}$. This is particularly relevant as we use tree-level and non-perturbative improvement [3] for the correlators. Lastly, we observe that the cutoff effects are strongly $r T$ and $T$ dependent. These issues necessitate either a fully global analysis in $r T, T$ and $a$ or subsequent interpolations in $r T$ and $T$

\footnotetext{
${ }^{1}$ For QCD this is simply $\left(L^{\mathrm{b}}\left(\beta, N_{\tau}\right)\right)^{2}$ since $L$ is real.
} 

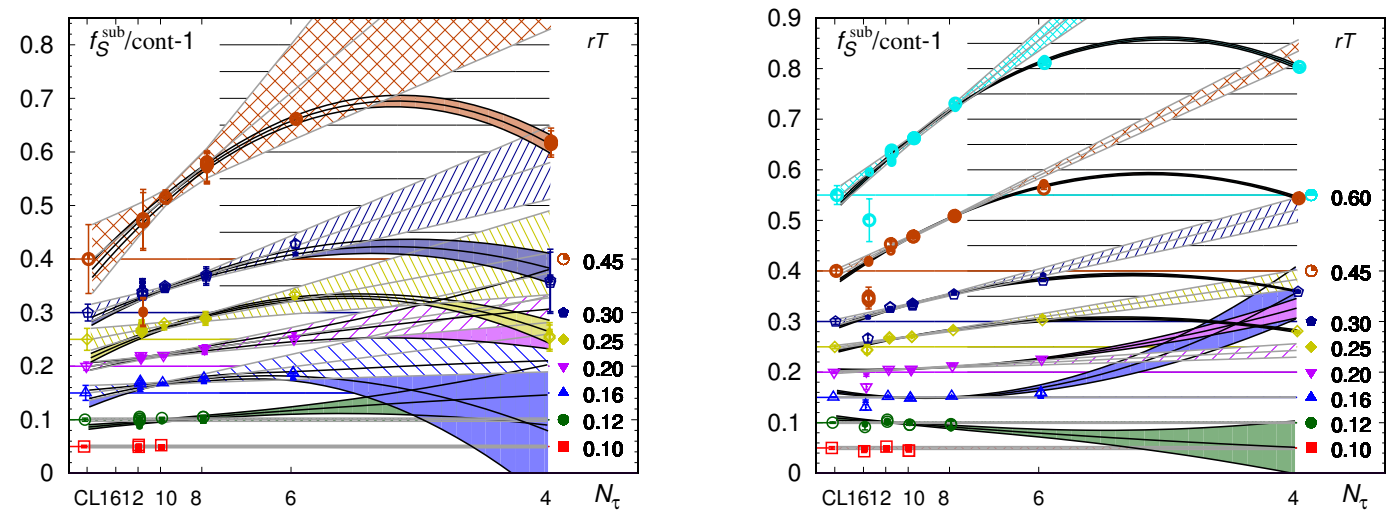

Figure 2: Scaling behavior of $F_{S}^{\text {sub }}$ for $T=200$ and $800 \mathrm{MeV}$ (from left to right). Larger open symbols indicate results from successive local interpolations, smaller filled symbols from global fits in $r T$ and $T$. Data have been normalized by the continuum limit and shifted vertically for better visibility. $F_{Q \bar{Q}}$ and $F_{S}$ follow the same overall trends in the scaling behavior.

followed by extrapolations in $a$ or $1 / N_{\tau}$ respectively. We pursue both approaches, performing first a full Jackknife analysis for each $F(T, a)$ to interpolate in $r T$ and then a temperature interpolation for each $F(r T, a)$ using smoothing splines with the bootstrap method for error propagation. Alternatively we perform global fits for each $F\left(N_{\tau}\right)$ restricted to overlapping low or high temperature intervals using the bootstrap method. As the results are largely commensurate we average over both and estimate systematic uncertainties from their difference. Eventually we extrapolate each $F^{\mathrm{sub}}(r T, T)$ to the continuum limit using different assumptions on scaling behavior, namely

$$
F^{\mathrm{sub}}\left(r T, T, N_{\tau}\right)=F_{0}^{\mathrm{sub}}(r T, T)+\frac{F_{2}^{\mathrm{sub}}(r T, T)}{N_{\tau}{ }^{2}}+\frac{F_{4}^{\mathrm{sub}}(r T, T)}{N_{\tau}{ }^{4}}
$$

with or without the quadratic and quartic terms and for different restrictions of the $N_{\tau}$ range. We generally find that discretization errors are more prominent for larger $r T$ and for lower temperatures as we show in Fig. 2. Data for $N_{\tau}=6$ are in the $a^{2}$ scaling regime except for $T<200 \mathrm{MeV}$ or $r T>0.3$, while data for $N_{\tau}=4$ always require $a^{4}$ scaling. In the range of $r T \leq 0.15$ and $T \geq 180 \mathrm{MeV}$ data for $N_{\tau} \geq 8$ are within uncertainties consistent with the continuum limit. We piece together the continuum result from appropriate continuum extrapolations for each $(r T, T)$ range and finally renormalize by adding $2 F_{Q}(T)$ to the continuum limit.

We show the continuum limit of $F_{S}$ and $F_{Q \bar{Q}}$ in Fig. 3. We observe for $T \gtrsim 300 \mathrm{MeV}$ that the free energies reach their asymptotic value $2 F_{Q}$ within errors. For separations up to $r T \lesssim 0.25$ the difference between the $T=0$ static energy $V_{S}$ and the $T>0$ singlet free energy $F_{S}$ is small compared to the uncertainty of the renormalization constant $2 C_{Q}$. For sufficiently small separations $F_{Q \bar{Q}}-T \ln (9)$ is numerically consistent with $F_{S}$ as suggested by the gauge-dependent color decomposition [9]

$$
C_{P}(r T, T)=\exp \left[-F_{Q \bar{Q}}(r T, T) / T\right]=\frac{1}{9} \exp \left[-F_{S}(r T, T) / T\right]+\frac{8}{9} \exp \left[-F_{A}(r T, T) / T\right] .
$$

\section{Vacuum-like regime}

It is clear that free energies depend only mildly on effects of the thermal medium at sufficiently 

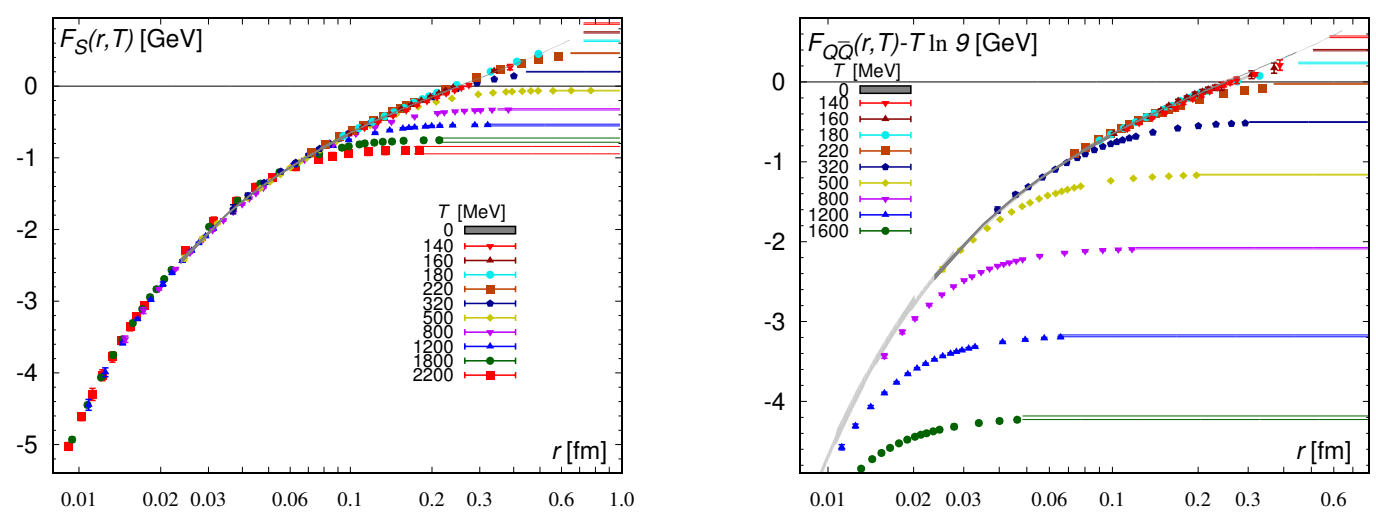

Figure 3: Continuum limit of $F_{S}$ (left) and $F_{Q \bar{Q}}$ (right). We show $V_{S}$ at $T=0$ as a dark gray band and $F_{S}$ for $r t<0.25$ as a light gray band. The colored lines indicate $2 F_{Q}$ (left) or $2 F_{Q}-T \ln 9$ (right) respectively.
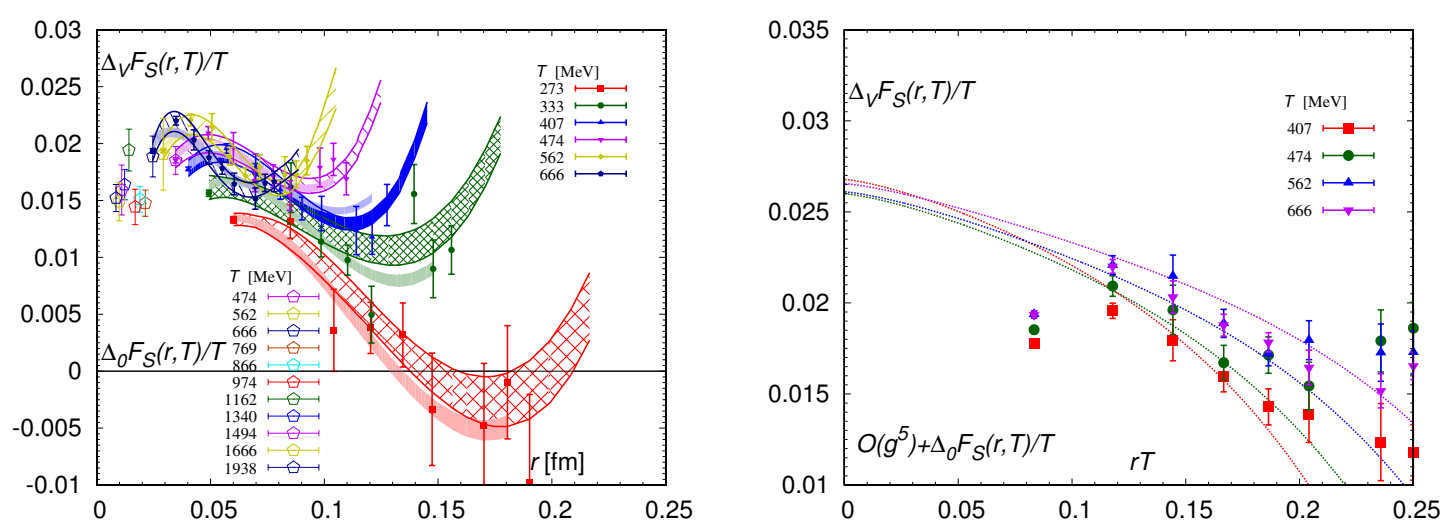

Figure 4: In the vacuum-like regime $\Delta_{V} F_{S}$ and $\Delta_{0} F_{S}$ are commensurable (left). Up to a shift of similar size as $\Delta_{0} F_{S}$ the $r T$ dependence is consistent with the pNRQCD prediction at $\mathscr{O}\left(g^{5}\right)$ shown as lines (right).

small separations. Thanks to asymptotic freedom small separations imply weak coupling. Thus, there may be a regime where weak coupling and lattice QCD results are compatible.

The difference $\Delta_{V} F_{S}=V_{S}-F_{S}$ is particularly suitable for a comparison between both approaches. Namely, the self-energy divergences in $\Delta_{V} F_{S}$ cancel exactly, thus alleviating the need for renormalization with $C_{Q}$ and the associated error. Moreover, as discretization errors for $r / a \lesssim 3$ due to the breaking of rotational symmetry in $V_{S}$ and $F_{S}$ are similar, $\Delta_{V} F_{S}$ benefits from a strong compensation of errors. $\Delta_{V} F_{S}$ has been calculated in pNRQCD up to $\mathscr{O}\left(\alpha_{s}^{3}\right)$ [8]. The result reads

$$
\Delta_{V} F_{S}(r T, T) \equiv V_{S}(r)-F_{S}(r T, T)=\alpha_{s}^{2} T\left[\Delta f_{g}(r T)+\Delta f_{f}(r T)+\Delta f_{s}(r T, g)\right]+\mathscr{O}\left(\alpha_{s}^{3}\right) .
$$

Gluonic, fermionic and screening terms $\Delta f_{g}, \Delta f_{f}$ and $\Delta f_{s}$ read as functions of $x=(\pi / 3) r T$

$$
\begin{aligned}
& \Delta f_{g}(x)=N_{c} C_{F}\left\{-\frac{1}{3} x+\frac{2 \zeta(3)}{\zeta(2)} x^{2}-\frac{22}{25} x^{3}\right\}+\mathscr{O}\left(\alpha_{s}, x^{4}\right), \\
& \left.\Delta f_{f}(x)=N_{f} C_{F}\left\{+\frac{3 \zeta(3)}{2 \zeta(2)} x^{2}-\frac{7}{10} x^{3}\right\}+\mathscr{O}\left(\alpha_{s}, x^{4}\right)\right), \\
& \Delta f_{s}(x)=-\frac{C_{F}}{\alpha_{s}}\left(\frac{m_{D}^{L O}}{T}\right)^{3} \frac{1}{4 \zeta(2)} x^{2}+\mathscr{O}\left(g^{3} x^{4}\right) .
\end{aligned}
$$


$m_{D}^{L O}=g T \sqrt{\left(N_{c}+N_{f} / 2\right) / 3}$ is the leading order Debye mass. The coefficients of $x^{2}$ in $\Delta f_{g}$ and $\Delta f_{f}$ on the one hand and $\Delta f_{s}$ on the other hand have opposite sign, indicating a compensation between different thermal effects.

We take note that on dimensional grounds $F_{S}$ may contain an $r T$ independent term ${ }^{2}$ of order $\alpha_{s}^{3} T$. We denote this term as $\Delta_{0} F_{S}(T)$ and determine it at the smallest available distance, $r / a=1$,

$$
\Delta_{0} F_{S}(T)=\lim _{a \rightarrow 0} \lim _{r \rightarrow 0}\left\{F_{S}(r, T, a)-\lim _{T \rightarrow 0} F_{S}(r, T, a)\right\} \approx\left\{F_{S}\left(\frac{1}{a}, T, a\right)-\lim _{N_{\tau} \rightarrow \infty} F_{S}\left(\frac{1}{a}, \frac{1}{a N_{\tau}}, a\right)\right\} .
$$

We calculate $\Delta_{0} F_{S}(T)$ for fixed lattice spacing $a$ through Eq. (4.5) using only $N_{\tau} \geq 10$ and find no systematic $a$ dependence. Thus, we consider $\Delta F_{S}(1 / a, T, a)$ as continuum estimate for $\Delta_{0} F_{S}(T)$. Using $N_{\tau}=12$ data we show in Fig. 4 the lattice results for $\Delta_{V} F_{S}$ and $\Delta_{0} F_{S}$ together in the left panel and with the weak-coupling results in the right panel. Below $r T \lesssim 0.25 \Delta_{V} F_{S}$ is small and only mildly $r T$ dependent, about $2 \%$ of the temperature, while above $r T \gtrsim 0.25$ temperature effects in $\Delta_{V} F_{S}$ rapidly become large, giving rise to a steep $r T$ dependence due to color screening.
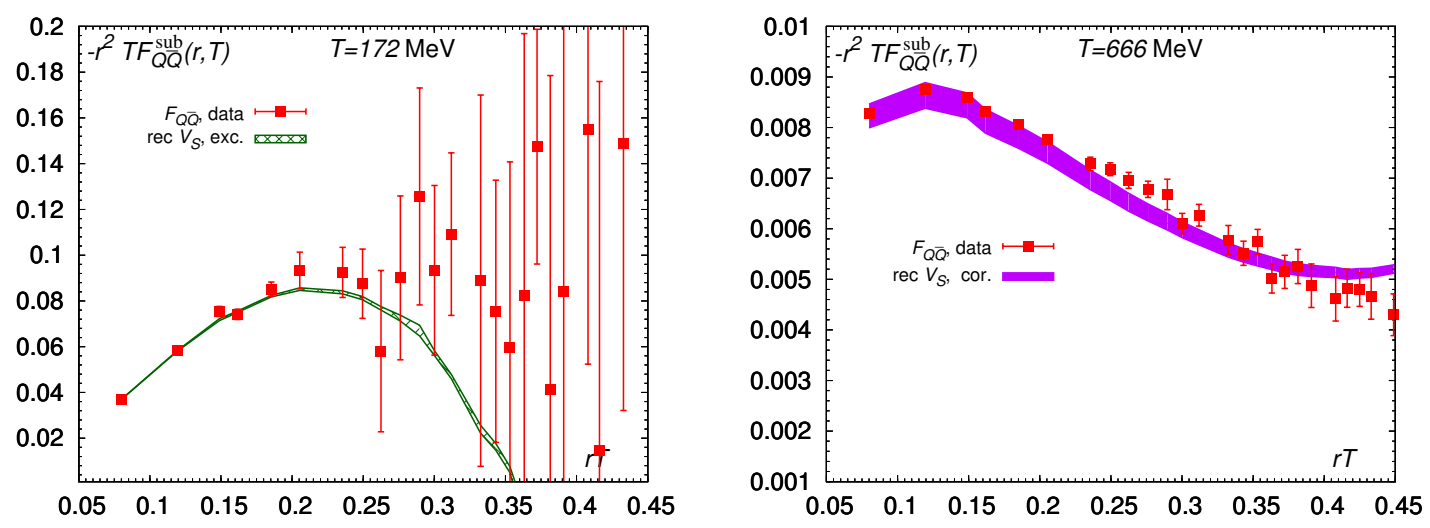

Figure 5: In the vacuum-like regime pNRQCD describes $F_{Q \bar{Q}}$ in terms of $V_{S}, V_{A}$ and $L_{A} . F_{Q \bar{Q}}$ is insensitive to $V_{A}$ and $L_{A}$ for $T<200 \mathrm{MeV}$ (left), while both and Eq. (4.7) must be included at high temperatures (right).

In weakly-coupled pNRQCD the Polyakov loop correlator satisfies at small separations a gauge-invariant decomposition in terms of singlet and adjoint free energies [10], which is reminiscent of Eq. (3.2). Up to higher orders in $r T$ and $r m_{D}$ this is a relation with singlet and adjoint potentials, $V_{S}$ and $V_{A}$, and the Polyakov loop in the adjoint representation, $L_{A}$. Namely,

$$
C_{P}(r T, T)=\exp \left[-F_{Q \bar{Q}}(r T, T) / T\right]=\frac{1}{N_{c}^{2}} \exp \left[-V_{S}(r) / T\right]+\frac{N_{c}^{2}-1}{N_{c}^{2}} L_{A}(T) \exp \left[-V_{A}(r) / T\right]
$$

We obtain the adjoint potential $V_{A}$ from $V_{S}$ by including the Casimir-scaling violation [11]

$$
\left(N_{c}^{2}-1\right) V_{A}+V_{S}=N_{c} \frac{N_{c}^{2}-1}{8} \frac{\alpha_{s}^{3}}{r}\left(\pi^{2} / 4-3\right)+\mathscr{O}\left(\alpha_{s}^{4}\right) .
$$

Via Casimir scaling we obtain $L_{A}=\left[\left(1-\delta_{8}\right) L_{F}\right]^{C_{F} / C_{A}}$. Casimir-scaling violations are parameterized by $\delta_{8}$, which is empirically compatible with zero for $T>300 \mathrm{MeV}$ [12]. Using $N_{\tau}=12$ data we show in Fig. 5 that $F_{Q \bar{Q}}$ is described well by Eq. (4.6) up to $r T \lesssim 1 / 3$. In particular, we do not

\footnotetext{
${ }^{2}$ The $r T$ independence is supposed to hold only for the leading order, i.e. $\alpha_{s}^{3}$.
} 
see significant differences between Eq. (4.6) including or excluding the $V_{A}$ contribution for $T<$ $200 \mathrm{MeV}$, indicating that the color-adjoint contribution is suppressed. This is contrasted by a poor reconstruction of $F_{Q \bar{Q}}$ at high temperatures without correction via Eq. (4.7). Hence, cancellation between color-singlet and -adjoint states becomes stronger with increasing temperature, or in other words, the spectral gap shrinks. This lowered gap suggests that real-time processes like dissociation and recombination may be dominant for compact heavy quark-antiquark systems in a thermal bath.

\section{Electric screening regime}

For $Q \bar{Q}$ separations larger than $r T \gtrsim 0.25$ the correlators invariably become more and more sensitive to the color screening. In this section we establish whether this can be related to the Debye screening in dimensionally-reduced effective field theories. These can be obtained for quantum field theories at sufficiently high temperatures by integrating out the non-static Matsubara modes through a UV cutoff [13]. The three-dimensional effective theory for QCD is called electrostatic QCD (EQCD) [14]. Its low-energy degree of freedom is the electrostatic gauge field, whose mass parameter corresponds to the perturbative Debye mass. Since the running of the gauge coupling in EQCD is far milder than for QCD, we anticipate a regime around $r m_{D} \sim 1$ with moderately weak interactions even at values of $r$ where confinement would be observable in the vacuum.

Both the free energy [9] and the singlet free energy [15] have been calculated in EQCD at NLO in terms of the Debye mass. For consistent counting we require $m_{D}$ determined at NLO [14],

$$
\left(m_{D}^{N L O}\right)^{2}=\left(1+\frac{g^{2}}{(4 \pi)^{2}}\left[\frac{5 N_{c}+2 N_{f}(1-4 \ln 2)}{3}+\frac{11-2 N_{f}}{3} L_{b}\right]\right)\left(m_{D}^{L O}\right)^{2}-C_{F} N_{f} \frac{g^{4}}{(4 \pi)^{2}},
$$

where $L_{b}$ is the bosonic Matsubara sum. Moreover we use the NLO field renormalization factor $Z_{1}$,

$$
Z_{1}=1+\alpha_{s}\left[\frac{11 N_{c}}{3}\left(L_{b}+1\right)-\frac{2 N_{f}}{3}\left(L_{f}-1\right)\right]
$$

with bosonic and fermionic Matsubara sums $L_{b}=2 \ln \left[\mu e^{\gamma_{E}} /(4 \pi T)\right]$ and $L_{f}=2 \ln \left[\mu e^{\gamma_{E}} /(\pi T)\right]$. $\alpha_{s}, m_{D}$ and $Z_{1}$ explicitly depend on the renormalization scale $\mu$. The NLO results for the subtracted free energies read

$$
\begin{aligned}
F_{S}^{\mathrm{sub}, \mathrm{NLO}}= & -C_{F} \frac{\alpha_{s}(\mu) e^{-r m_{D}(\mu)}}{r}\left(Z_{1}^{2}(\mu, T)\right. \\
& \left.+\alpha_{S}(\mu) N_{c} r T\left[2-\ln \left(2 r m_{D}(\mu)\right)-\gamma_{E}+e^{+2 r m_{D}(\mu)} E_{1}\left(2 r m_{D}(\mu)\right)\right]\right) \\
F_{Q \bar{Q}}^{\mathrm{sub}, \mathrm{NLO}}= & \left\{\frac{\alpha_{s}(\mu) e^{-r m_{D}(\mu)}}{3 r T}\right\}^{2}\left(Z_{1}^{2}(\mu, T)+2 N_{c} \alpha_{S}(\mu) r T\left[\frac{3-\gamma_{E}}{2}+\frac{\ln \left[r m_{D}(\mu)\right]}{2}-\ln \left[2 r m_{D}(\mu)\right]\right.\right. \\
& \left.\left.+\frac{\ln \left[2 r m_{D}(\mu)\right]+\gamma_{E}}{r m_{D}(\mu)}-\frac{1}{2} g\left[2 r m_{D}(\mu)\right]+\frac{1}{2} h\left[4 r m_{D}(\mu)\right]-\frac{h\left[2 r m_{D}(\mu)\right]}{r m_{D}(\mu)}\right]\right)
\end{aligned}
$$

where the functions $g(y)$ and $h(y)$ are defined as

$$
g(y)=\int_{0}^{\infty} d x \frac{e^{-x y}}{x+1} \ln \left[\frac{x+2}{x}\right], \quad h(y)=e^{y} E_{i}(-y) .
$$



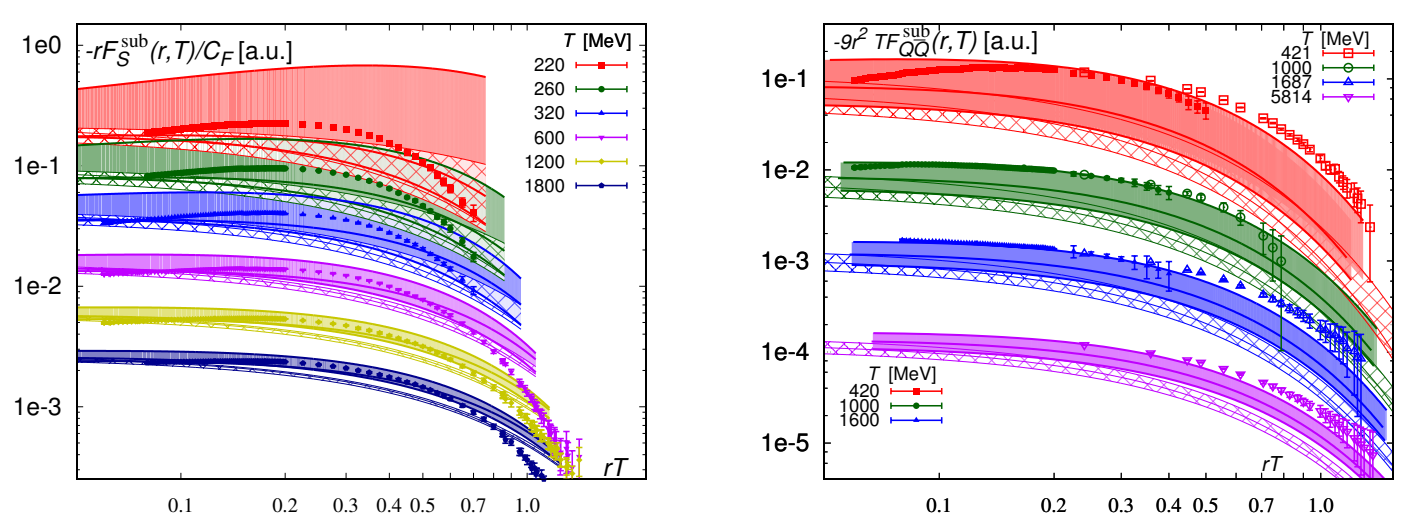

Figure 6: Subtracted free energies from lattice and EQCD. Hashed or solid bands represent the EQCD results at LO or NLO respectively for a variation of the cutoff scale as $\mu=\pi T, 2 \pi T$ and $4 \pi T$. For $F_{Q \bar{Q}}$ we show continuum results as filled symbols and $N_{\tau}=4$ results with $N_{\sigma} / N_{\tau}=6$ as open symbols (right).

The second power of first term in Eq. (5.4) is due to the cancellation between color-singlet and color-adjoint contributions discussed already in Eq. (4.6).

We show continuum-extrapolated lattice results for the subtracted free energies together with the EQCD predictions at LO and NLO in Fig. 6. The lattice results are much better described by EQCD at NLO than at LO, indicating that the weak-coupling expansion is indeed on solid footing in the electric screening regime. For small $(r T \ll 0.2)$ and large $(r T \gg 0.4)$ separations the $r T$ dependence of $F_{S}$ is not consistent with EQCD. We understand the former as the vacuumlike regime, for which pNRQCD provides an appropriate description, cf. Sec. 4. We understand the latter as the regime of asymptotic screening. The corresponding asymptotic screening mass is considerable larger than the Debye mass of EQCD. At small $r T$ we see a non-EQCD regime in $F_{Q \bar{Q}}$, too. The $r T$ dependence in this regime, which rapidly becomes shorter with increasing temperature, is inconsistent with EQCD. From Sec. 4 we understand that the spectral gap is still large in this vacuum-like regime. With available lattice data we cannot resolve the onset of a regime of asymptotic screening in $F_{Q \bar{Q}}$.

\section{Asymptotic screening regime}

In the asymptotic regime screening is dominated by the magnetostatic scale $g^{2} T$. Whereas $g^{2} T$ is formally smaller than the electrostatic Debye mass scale $g T$, it turns out to be more important numerically for any physically relevant values of the temperature and the gauge coupling. The magnetic mass $m_{M} \sim g^{2} T$ in turn leads to a breakdown of weak-coupling power counting in diagrammatic descriptions [9]. As a consequence, this regime is inherently non-perturbative.

Long range interactions contributing to the free energies are dominated by one-particle-exchange processes, thus giving rise to a Yukawa-type interaction $\sim \exp [-M r] / r$ for a mode of mass $M$. In the case of $C_{P}$ these OPE processes concur in some intermediate regime (between electrostatic and magnetostatic regimes) by two-particle-exchange processes $\sim\{\exp [-M r] / r\}^{2}$.

For multiple reasons we cannot simply study asymptotic screening through a straightforward fit with Yukawa potential at large values of $r$. First we have to account for finite volume corrections 

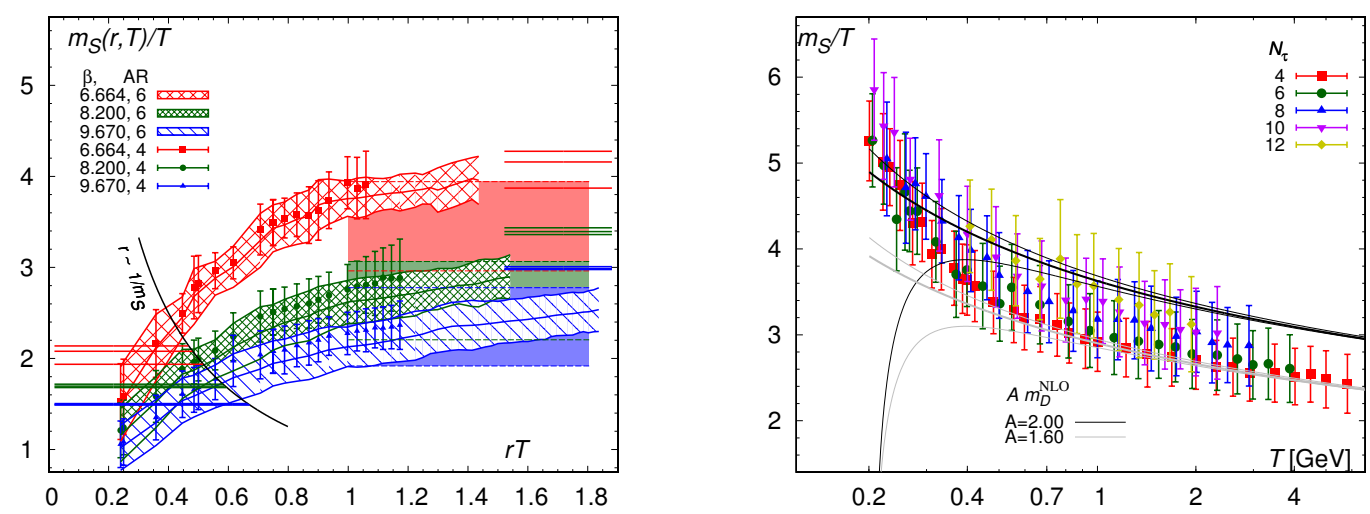

Figure 7: Asymptotic screening mass for $F_{S}$. We observe no statistically significant volume dependence of $m_{S} / T$, which is for $r \sim 1 / m_{s}$ close to the NLO Debye mass $m_{D}$ (horizontal lines from left). $m_{S} / T$ stays somewhat below $2 m_{D} / T$ (horizontal lines from right) in the accessible range for $N_{\tau}=4$ (left). The corrected values of $m_{S} / T$ are shown as solid bands in the left panel and as function of $T$ in the right panel.

since cancellations in Eqs. (2.5) and (2.6) are exact only in infinite volume and may be limited by statistics. In fact we see a volume dependence by comparing screening functions $S_{1}=-r F^{\text {sub }}$ with aspect ratios $N_{\sigma} / N_{\tau}=6$ and $N_{\sigma} / N_{\tau}=4$. Volume dependence is reduced for larger ensembles and we successfully model it as a constant offset that we subtract from correlators to bring results for different aspect ratios into numerical agreement. Second we cannot determine the asymptotic screening mass through a straightforward fit in the far asymptotic regime due to an exponential drop of the signal-to-noise ratio. Instead we have to start fitting at intermediate $r T$ values for which the system is not necessarily beyond the electric screening regime. Hence, we fit $F_{S}^{\text {sub }}$ and $F_{Q \bar{Q}}^{\text {sub }}$ as

$$
F^{\mathrm{sub}}(r T, T, a)=A \frac{e^{-M R}}{R}+C, R \equiv r T
$$

and vary both small $\left(R_{\min }\right)$ and large $\left(R_{\max }\right)$ ends of the fit range to obtain a local definition of the screening mass $M=m / T$.

In the case of $F_{S}$ the parameter $M\left(R_{\min }, R_{\max }\right)$ is a monotonically rising function of $R_{\min }$ and $R_{\max }$ that becomes considerably flatter for $R_{\min } \gg 0.7$. Since the dependence on $R_{\max }$ is rather mild we average for fixed $R_{\min }$ over all $R_{\max }$ that permit good fits for Eq. (6.1) and estimate a systematic uncertainty from the spread. Even for $F_{S}^{\text {sub }}$ with $N_{\sigma} / N_{\tau}=6$ we still observe a non-negligible increase even for the largest $R_{\min }$, cf. left panel of Fig. 7. For $N_{\sigma} / N_{\tau}=4$ we are not even able to obtain a stable screening mass for $R_{\min } \gg 0.7$ in many cases. For $R_{\min } \sim 1 / m_{D}$ the screening mass is close to the NLO Debye mass, while for $r T \gg 1$ the screening mass remains below twice the NLO Debye mass. We estimate a temperature independent additive correction between the parameter $M$ for $R_{\min }=0.5$ and for $R_{\min } \gtrsim 1.0$ and take the corrected result as our estimate of the asymptotic screening mass. We plot this corrected value of the screening mass as function of the temperature in the right panel of Fig. 7. Although there is a trend to larger values of $M$ towards the continuum limit, screening masses for all $N_{\tau}$ are numerically commensurable. The temperature dependence for $T>400 \mathrm{MeV}$ and the $N_{f}$ dependence are consistent with the NLO Debye mass.

In the case of $F_{Q \bar{Q}}$ the parameter $M\left(R_{\min }, R_{\max }\right)$ exhibits no statistically significant dependence on either $R_{\min }$ and $R_{\max }$ beyond $R_{\min } \gtrsim 0.5$. For $N_{\tau}=4$ and $N_{\sigma} / N_{\tau}=6$ we find a monotonic 


\begin{tabular}{|c|c|c|c|}
\hline$T[\mathrm{MeV}]$ & 421 & 1687 & 5814 \\
\hline$m_{Q \bar{Q}} / T$ & $4.6(1)$ & $4.2(3)$ & $3.9(2)$ \\
\hline
\end{tabular}

Table 1: The screening mass of $F_{Q \bar{Q}}$ for $N_{\tau}=4$ and $N_{\sigma} / N_{\tau}=6$ for $R_{\min }=0.5$.

decrease of $m_{Q \bar{Q}} / T$ with temperature, see Tab. 1 .
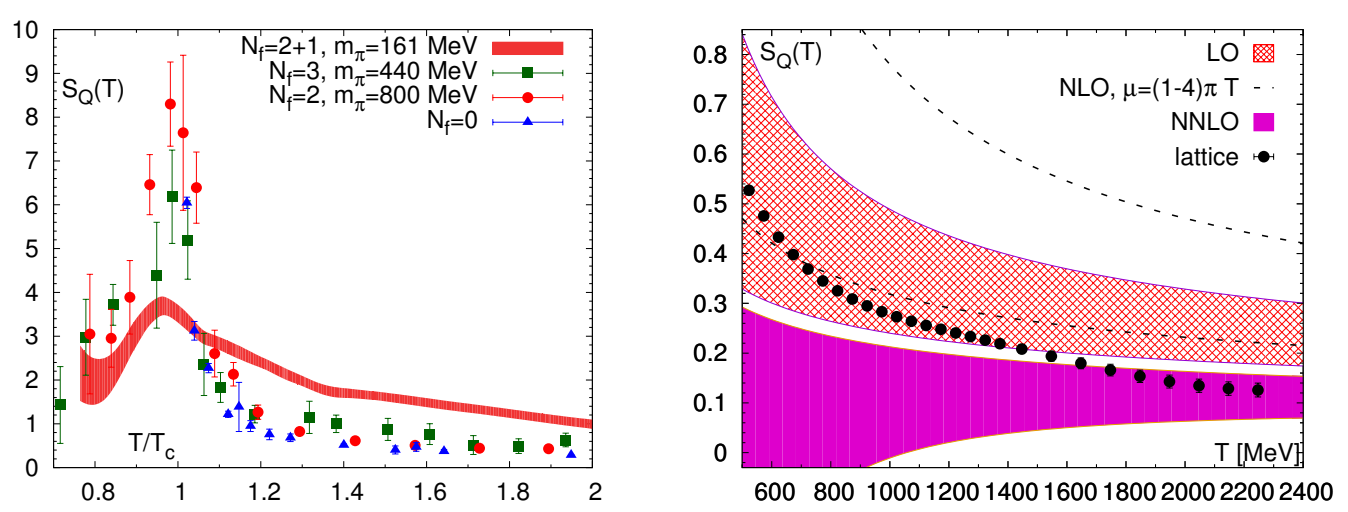

Figure 8: The entropy $S_{Q}$ of a static quark is sensitive to the color screening and deconfinement.

Asymptotically separated quarks eventually decouple completely and any quark-antiquark free energy approaches $2 F_{Q}$. We study this asymptotic regime in terms of the entropy of a static quark,

$$
S_{Q}=-\frac{d F_{Q}}{d T}
$$

which is renormalization-scheme independent in the continuum limit. As the entropy is at leading order $S_{Q}^{\mathrm{LO}}=C_{F} \alpha_{S}(\mu) m_{D}^{\mathrm{LO}}(\mu) /(2 T)$, it is a direct probe of the color screening properties of the thermal QCD medium. $S_{Q}$ is peaked in the crossover region and the peak position $T_{S}\left(N_{\tau}\right)$ is numerically consistent with the peak position $T_{\chi}\left(N_{\tau}\right)$ of the chiral susceptibilities on the same set of ensembles. This suggests that the critical temperatures related to the phase transitions in the opposite corners of the Columbia plot are smoothly connected, since the entropy and chiral susceptibilities are primarily sensitive to different aspects of the crossover transition. For lower quark masses or larger $N_{f}$, the peak of $S_{Q}$ is less pronounced, cf. the left panel of Fig. 8, suggesting that the peak may indeed be washed out completely for ensembles close to the chiral limit.

The Polyakov loop and the entropy have been calculated up to NNLO [16]. We have made an order-by-order comparison to lattice results with $N_{\tau}=4$ [6], seeing remarkably poor convergence at NLO. This can be understood as a consequence of the dominance of the static Matsubara mode in $S_{Q}$. First indications of consistency between NNLO and lattice results for $N_{\tau}=4$ were seen for $T \gtrsim 3 \mathrm{GeV}$. With the new ensembles we extend the continuum limit to $T \gtrsim 2 \mathrm{GeV}$ and find consistency with NNLO already for much lower temperatures, $T \gtrsim 1.7 \mathrm{GeV}$ as shown in the right panel of Fig. 8.

\section{Conclusions}

We study color screening in the quark-gluon-plasma with lattice QCD and weak-coupling approaches. We extract the continuum limit of the Polyakov loop correlator and related quantities 
in an unprecedented range up to $T \gtrsim 2 \mathrm{GeV}$ and perform detailed comparisons to different effective field theory results at weak coupling. We successfully identify a vacuum-like regime, an electric screening regime and an asymptotic regime and establish a direct connection to the weak-coupling calculations and the realization of the assumed hierarchies of scales.

\section{Acknowledgments}

We acknowledge the support by the Bundesministerium für Bildung und Forschung (BMBF) under grant "Verbundprojekt 05P2015 - ALICE at High Rate (BMBF-FSP 202) GEM-TPC Upgrade and Field theory based investigations of ALICE physics" under grant No. 05P15WOCA1. The simulations have been carried out on the computing facilities of the Computational Center for Particle and Astrophysics (C2PAP) and Supermuc. The data analysis was performed using the R statistical package.

\section{References}

[1] A. Bazavov, PoS LATTICE 2014, 392 (2015).

[2] P. Petreczky, J. Phys. G 39, 093002 (2012) [arXiv:1203.5320 [hep-lat]].

[3] Preprint TUM-EFT 81/16: A. Bazavov, N. Brambilla, P. Petrèczky, A. Vairo, J. H. Weber, "Color screening in 2+1 Flavor QCD”.

[4] A. Bazavov et al., Phys. Rev. D 85, 054503 (2012) [arXiv:1111.1710 [hep-lat]].

[5] A. Bazavov et al. [HotQCD Collaboration], Phys. Rev. D 90, 094503 (2014) [arXiv:1407.6387 [hep-lat]].

[6] A. Bazavov, N. Brambilla, H.-T. Ding, P. Petreczky, H.-P. Schadler, A. Vairo and J. H. Weber, Phys. Rev. D 93, no. 11, 114502 (2016) [arXiv:1603.06637 [hep-lat]].

[7] A. Bazavov, P. Petreczky and J. H. Weber, arXiv:1710.05024 [hep-lat].

[8] M. Berwein, N. Brambilla, P. Petreczky and A. Vairo, Phys. Rev. D 96, no. 1, 014025 (2017) [arXiv:1704.07266 [hep-ph]].

[9] S. Nadkarni, Phys. Rev. D 33, 3738 (1986).

[10] N. Brambilla, J. Ghiglieri, P. Petreczky and A. Vairo, Phys. Rev. D 82, 074019 (2010) [arXiv:1007.5172 [hep-ph]].

[11] B. A. Kniehl, A. A. Penin, Y. Schroder, V. A. Smirnov and M. Steinhauser, Phys. Lett. B 607, 96 (2005) [hep-ph/0412083].

[12] P. Petreczky and H.-P. Schadler, Phys. Rev. D 92, no. 9, 094517 (2015) [arXiv:1509.07874 [hep-lat]].

[13] E. Braaten and A. Nieto, Phys. Rev. D 51, 6990 (1995) [hep-ph/9501375].

[14] E. Braaten and A. Nieto, Phys. Rev. D 53, 3421 (1996) [hep-ph/9510408].

[15] Y. Burnier, M. Laine and M. Vepsalainen, JHEP 1001, 054 (2010) Erratum: [JHEP 1301, 180 (2013)] [arXiv:0911.3480 [hep-ph]].

[16] M. Berwein, N. Brambilla, P. Petreczky and A. Vairo, Phys. Rev. D 93, no. 3, 034010 (2016) [arXiv:1512.08443 [hep-ph]]. 